\section{Anaphylaxie ist teuer}

\section{Schwere anaphylaktische Reaktionen sind lebensbedrohliche Erkrankungen. Die durch sie verursachten ökonomischen Belastungen wurden jetzt in Frankreich abgeschätzt.}

A naphylaxien treten bei $1-3 / 10.000$ Menschen im Jahr auf. Ihre Letalität wird auf $1 \%$ geschätzt. Nach einer britischen Studie haben sich die stationären Aufnahmen wegen einer anaphylaktischen Reaktion von 1990 bis 2007 versiebenfacht. Französische Ärzte werteten jetzt die Daten von 402 Patienten mit bestätigter anaphylaktischer Reaktion aus, um die damit verbundene volkswirtschaftliche Belastung abschätzen zu können.

Das klinische Bild umfasste ernste systemische Reaktionen mindestens zweier Organsysteme, massives Asthma bronchiale, Larynxschwellung mit Atmungsbehinderung sowie Schock mit kardiovaskulärem Kollaps. Als Kosten wurden die direkten medizinischen Kosten (Medikamente, Konsultationen, sonstige stationäre Kosten) sowie die direkten nicht medizinischen Kosten (Transport etc.) berechnet. Dazu kamen die indirekten Kosten wie Abwesenheit des Patienten oder der Eltern vom Arbeitsplatz oder der Schule.

Insgesamt ergaben sich durchschnittliche Gesamtkosten je nicht letalem Anaphylaxiefall von 1.895 Euro bei Reaktionen gegen Nahrungsmittel oder Medikamente und von 4.053 Euro bei einer Insektengiftanaphylaxie. Die direkten Kosten reichten von 75 Euro für eine kurze Behandlung bei bekannter Allergie bis hin zu 4.445 Euro bei schwerer Anaphylaxie mit notfallmedizinischem Ein- satz, Aufenthalt auf der Intensivstation und aufwändigen Allergietests. Die durchschnittlichen indirekten Kosten lagen bei 315 Euro, entsprechend einer Abwesenheit vom Arbeitsplatz von drei Tagen. Insgesamt schätzen die Autoren nach diesen Daten die jährlich in Frankreich entstehenden Kosten durch Anaphylaxien auf rund 4,8 Millionen Euro.

Fazit: Die Kosten einer anaphylaktischen Reaktion wurden in dieser Studie mit insgesamt 1.895 Euro (Nahrungs- und Medikamentenallergie) bzw. 4.053 Euro (Hymenopterengiftallergie) angegeben. Diese Kosten sind nach Meinung der Autoren eher unterschätzt, da beispielsweise stationär auftretende Anaphylaxien nicht berücksichtigt wurden.

Flabbee J et al. The economic costs of severe anaphylaxis in France: an inquiry carried our by the Allergy Vigilance Network. Allergy 2008; 63:360-65

\title{
Welcher Naturlatexextrakt ist der beste?
}

\section{Für die Diagnostik einer Naturlatexallergie stehen verschiedene Extrakte zur Auswahl, deren Sensitivität und Spezifität erstmals direkt miteinander verglichen wurden.}

M öglicherweise erfassen naturbelassene Naturlatexextrakte nicht alle Sensibilisierungen, denn die meisten naturlatexhaltigen Produkte werden aus Latexmasse hergestellt, der

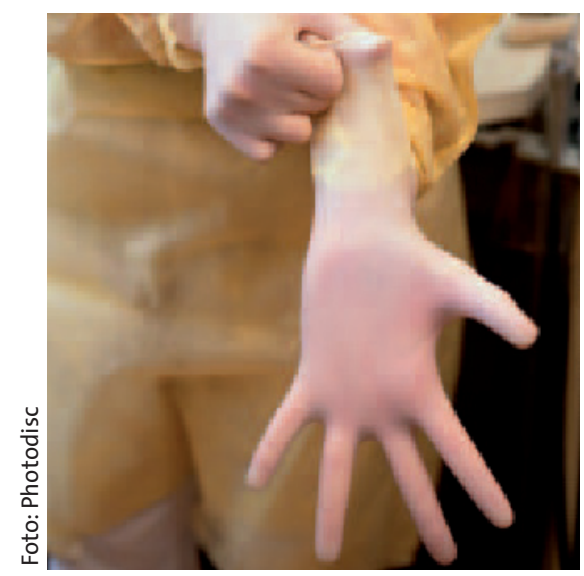

Die Naturlatexallergie ist immer noch ein Problem, die Testung mit Naturlatex auch. aus verarbeitungstechnischen Gründen Ammoniak $\left(\mathrm{NH}_{3}\right)$ zugesetzt wurde. Die Alkalisierung könnte zu einer Veränderung der Allergenzusammensetzung führen.

42 Kinder und Jugendliche mit Verdacht auf Naturlatexallergie wurden deshalb in einer prospektiven Studie ausführlich anamnestisch befragt. Außerdem wurden das naturlatexspezifische Serum-IgE bestimmt (Unicap RAST, positiv bei $>0,7 \mathrm{kU} / \mathrm{l}$ ) und ein offener Handschuhprovokationsrest durchgeführt. 16 Patienten hatten bereits mehrere Operationen hinter sich, bei 13 lag eine Allergie gegen Früchte und/oder Gemüse vor. Für die Hautpricktests wurden zwei nicht mit $\mathrm{NH}_{3}$ behandelte (Produkt A: Lofarma; Produkt B: Stallergenes) und ein mit $\mathrm{NH}_{3}$ behandelter Naturlatexextrakt (Produkt C : ALK-Abellò) verwendet. Als posi- tives Ergebnis galt ein Quaddeldurchmesser $>3 \mathrm{~mm}$. Bei den Kindern mit einer gesicherten Naturlatexallergie traten im Pricktest mit $\mathrm{NH}_{3}$-behandeltem Extrakt durchschnittliche Quaddelgrößen von 7,3 mm, bei Verwendung der beiden nicht mit $\mathrm{NH}_{3}$ behandelten Extrakten Quaddeldurchmesser von 5,7 bzw. 4,4 mm auf ( $\mathrm{p}=0,004$ für $\mathrm{C}$ gegenüber $A$ und $B$ ). Die Sensitivitäten lagen bei $65 \%$ (A), 81\% (B) und 96\% $(\mathrm{C} ; \mathrm{p}=0,0084 \mathrm{C}$ gegenüber $\mathrm{A})$. Bei der diagnostischen Spezifität bestanden keine signifikanten Unterschiede (94\%, $88 \%$ und $94 \%$ für $\mathrm{A}, \mathrm{B}$ und $\mathrm{C}$ ).

Fazit: Die Hauttestung kann je nach Naturlatexextrakt unterschiedlich deutlich ausfallen. Bei Kindern mit Naturlatexallergie hat ein ammoniakbehandelter Naturlatexextrakt eine höhere Sensitivität als nicht behandelte Extrakte. $b k$

Bernardini R et al. Sensitivity and specificity of different skin prick tests with latex extracts in pediatric patients with suspected natural rubber latex allergy a cohort study. Pediatr Allergy Immunol 2008; 19:315-8 